\title{
Ultrasound-guided transversus Abdominis plane block for postoperative analgesia after abdominal sugery by using bupivacain alone and bupivacain with additive
} M.Y.Serry, E.S.Abd Elazym, D.H.El Berbary and A.M.Hassan

Anesthesiology and ICU department, Dept., Faculty of Medicine, Benha Univ., Benha, Egypt E-Mail:samoaaa8@gmail.com

\begin{abstract}
Background: Caesarean section is often linked with moderate to severe pain, resulting in patient discontent, patient rehabilitation, and lengthy hospitalisation. This research aimed to assess and evaluate the effectiveness and safety of dexmedetomidine and dexamethasone in addition for postoperative analgesia in patients in caesarean section with bupivacaine in the ultrasound guided TAP block. Methods: Random allocation of 90 patients to three equal groups: Group A Transversal Ultrasound Controlled Abdominal Fluke (uTAP):) got bilateral transversal ultrasound guided ultrasound plane block. $20 \mathrm{ml}$ of 0.25 percent bupivacaine and $2 \mathrm{ml}$ of normal saline were administered on both sides. Group B transversal guided ultrasound (UTAP): Bilateral ultrasound guided transversal plane block (UTAP). Dexmedetomidine $1 \mathrm{mcg} / \mathrm{kg}$ was dissolved into $2 \mathrm{ml}$ of normal saline and 0,25 percent of the amount was added on each side to $20 \mathrm{ml}$ of bupivacaine. Group C Transversal Guided Abdominal Block (uTAP): got the bilateral ultrasound transversal guided plane block of the abdominal abdominal ultrasonography. Dexamethasone $4 \mathrm{mg}$ had been dissolved by adding $20 \mathrm{ml}$ of bupivacaine to $2 \mathrm{ml}$ of normal saline. 25 per cent. Results and Conclusion: adding dexmedetomidine to the TAP block for TAP substantially reduces the VAS value, reduces the usage of narcotic in the first 12 hours, increases drowsiness and extends TFA more than dexamethasone.
\end{abstract}

Keywords: Transversus Abdominis, Block, Analgesia, Abdominal sugery, Bupivacaine.

\section{Introduction}

Caesarean section is the world's most common operation. In the first 24 hours after surgery, patients suffer moderate to severe discomfort. The research was designed to assess the effectiveness of dexmedetomidine and dexamethasone with ultrasound guided TAP block bupivacaine for post-operative analgesia in caesarean patients. Caesarean section is frequently linked with moderate to severe discomfort that may lead to patient unhappiness, poor recovery and extended hospitalisation. A well-planned analgesic scheme is thus needed to provide sufficient mother satisfaction, early breast feeding and early movement to avoid thrombosis risk owing to pain immobility. Somatic pain (abdominal wall incision and less due to visceral discomfort) is mainely affected by pain after caesarean section (uterus). A multimodal strategy for the treatment of postoperative pain in the form of NSAIDS, opioids, local LA infiltrations and neuraxial blocks in the form of epidurals is necessary. [1] Airplane block is used to give anterior and lateral abdominal wall analgesia. A local anaesthetic (Bupivacaine) solution is injected into the face of the inner and transversal oblique muscles under ultrasound supervision. The procedural ease of this block along with the dependable degree of analgesia (T6-L1) and extended duration and quality of analgesia make TAP a viable choice for lower abdominal gyneecology operations (ASRA). [2]

TAP block has been shown to decrease postoperative usage of opioids, extend the time to first apply for additional analgesia and offer more effective pain management while reducing adverse effects linked to opioids, including drowsiness and postoperative nausea and vomiting. It is of crucial significance to provide adequate postoperative analgesia to enable early ambulation and avoid postoperative morbidity.
The analgesic system must achieve the objectives of safe, efficient analgesics with minimum adverse effects [3].

Many additions for the duration of analgesia for peripheral nerve blocks, including epinephrine, buprenorphine, tramadol, sodium bicarbonate, dexmedetomidine, fentanyl, ketamine and neostigmine, have been investigated in local anaesthetics. Dexmedetomidine is a lipophilic $\alpha 2$ agonist derivative with a greater $\alpha 2$ receptor affinity. It has sedative, analgesic and sympatholitic actions, which stop numerous cardiovascular responses in the perioperative phase from occurring. The addition of dexmedetomidine to bupivacain in the TAP block leads to improved local anaesthetic and better pain management after surgery, without significant side effects. [4]

Dexamethasone is a systemically active glucocorticoid.

Added in peripheral blocks as an adjuvant to local anaesthetics, this prolongs analgesic duration. Action mechanism may be achieved with anti-inflammatory effects, increased local efficiency and absorption slowdown[5].

This research aimed to assess and evaluate the effectiveness and safety of dexmedetomidine and dexamethasone in addition for postoperative analgesia in patients in caesarean section with bupivacaine in the ultrasound guided TAP block.

\section{Patients and methods}

The study protocol was approved by the institutional ethical committee of Benha university hospitals. Informed patient written consent was obtained before enrolment in the study. 


\subsection{Type of Study}

Prospective, single blind randomized clinical study.

\subsection{Methods of randomization}

Patients were randomized into three equal groups. An online randomization program was used to generate random number list. Patient randomization numbers were concealed in opaque envelops which were opened by the study investigator.

\subsection{Methods of blindness}

Members of the study group involved in obtaining functional data were blinded to randomization for the period of data acquisition and analysis.

\subsection{Inclusion Criteria}

- ASA physical status: I, II and III.

- from the age of 18 to 45 years.

- Type of operations: Patients undergoing elective caesarean section by pfannenstiel incision.

\subsection{Groups allocation}

90 Patients were randomly allocated into three equal groups:

- Group A ultrasound guided transversus abdominis plane block (uTAP):): received bilateral ultrasound guided transversus abdominis plane block. $20 \mathrm{ml}$ of bupivacaine $0.25 \%$ and $2 \mathrm{ml}$ of normal saline was given on each side.

- Group B ultrasound guided transversus abdominis plane block (uTAP): received bilateral ultrasound guided transversus abdominis plane block. Dexmedetomidine $1 \mathrm{mcg} / \mathrm{kg}$ was dissolved in $2 \mathrm{ml}$ of normal saline and added to 20 $\mathrm{ml}$ of bupivacaine $0.25 \%$ was given on each side.

- Group C ultrasound guided transversus abdominis plane block (uTAP): received bilateral ultrasound guided transversus abdominis plane block. Dexamethasone $4 \mathrm{mg}$ was dissolved in $2 \mathrm{ml}$ of normal saline and added to $20 \mathrm{ml}$ of bupivacaine $0.25 \%$.

\subsection{Exclusion Criteria}

- Patient refusal

- patients with a history of cardiac, respiratory, renal or hepatic failure, coagulation disorders

- local infection at the site of block

- psychological disorders

- allergy to study medications and chronic use of pain medications or adrenoreceptors agonists or antagonists are excluded from this study.

\subsection{Preoperative visit}

One day before surgery all patients were interviewed to explain visual analogue scale (VAS) (figure 4-1). Also routine investigations in the form of twelve leads electrocardiography (ECG), complete blood count (CBC), coagulation profile (bleeding time, prothrombine time, international normalized ratio and partial thromboplastine time), liver functions, and kidney functions were fulfilled.

The main goal of the study is to compare the three groups with regards to the VAS pain scores, whereas secondary outcome measures included the time to first request for morphine (which is defined as the time elapsed between the injection of the local anesthetic and the first time for the patient to request for analgesia), 24-h morphine consumption, and side effects associated with morphine (sedation and nausea). All data are collected by a nurse who is blinded to the anesthetic technique.

\subsection{Measurements}

- Visual analogue pain score (VAS): is measured at rest, and on movement (knee flexion) at PACU, 2hrs, 4hrs, $8 \mathrm{hrs}, 12 \mathrm{hrs}$, and $24 \mathrm{hrs}$. Postoperative (VAS; where $0=$ no pain and $10=$ worst imaginable pain)

- Total morphine consumption in the first 24 hrs and Time to first analgesia request where recorded from the completion of TAP block to first given morphine dose.

\section{- TAP block performance time}

- Vital signs: heart rate (HR), mean arterial blood pressure (MABP), and respiratory rate (RR) are recorded at PACU, 2hrs, 4hrs, 8hrs, 12hrs, and 24hrs postoperative.

- Rescue analgesia plan will be given for VAS $\geq 5$ using morphine. Time of the first rescue analgesia together with frequency and total dose of the given drug through the 24 hours will be recorded.

- Comparison will be done2,4, 6, 12, and 24 postoperative

\subsection{Complications}

- Postoperative nausea and vomiting aone, $1=$ nausea, 2 = retching, 3 = vomiting). IV Metoclopramide $10 \mathrm{mg}$ bolus is offered for any patient with a score.

- Sedation is measured by using $(0=$ awake and alert, 1 = quietly awake, 2 = asleep but easily roused, 3 = deep sleep).

- Purities.

- Complications related to the block technique. The study ended 24 hours after the operation.

\subsection{Statistical methods}

Data management and statistical analysis were done using SPSS vs.25. (IBM, Armonk, New York, United States). Numerical data were summarized as means and standard deviations or medians and ranges. Categorical data were summarized as numbers and percentages. Comparisons between both groups were done using one-way ANOVA or Kruskal Wallis test for normally and non-normally distributed numerical data, respectively. Categorical data were compared using the Chi-square test. Post hoc analyses were done in case of a significant overall effect. All post hoc comparisons were Bonferroni adjusted. All $\mathrm{P}$ values ere two-sided. $\mathrm{P}$ values less than 0.05 were considered significant 


\section{Results}

As regard patient demographic data, There were no significant differences between three groups, including age, duration of surgery, weight height. These data are shown in the table 1

At different follow-up times, $\mathrm{SPO}_{2}$ didn't show any significant difference between the three groups. These data are shown in the table 2

The mean heart rate in group A was significantly higher than group B, with no significant differences between groups A \& $\mathrm{c}$ and groups B \& C. These data are shown in fig. (1).
Mean respiratory rate at baseline was $12 \mathrm{c} / \mathrm{m}$ in all groups and didn't show any change at different times in all groups.

Mean systolic blood pressure showed an overall significant difference between the three groups, at 15 minutes, it was significantly higher in group $\mathrm{C}$ than in group A and group B. Also, at 30, 45, and 60 minutes, mean systolic blood pressure showed an overall significant difference between the three groups. It was significantly higher in group C compared to group B at these times, fig. (2)

Table (1) Demographic data in the study groups.

\begin{tabular}{lccccccc}
\hline & \multicolumn{2}{c}{ Group A $(\mathbf{n}=\mathbf{3 0})$} & \multicolumn{2}{c}{ Group B $(\mathbf{n}=\mathbf{3 0})$} & \multicolumn{2}{c}{ Group C $(\mathbf{n}=\mathbf{3 0})$} & \multicolumn{2}{c}{ ) } \\
& Mean & $\mathbf{\pm S D}$ & Mean & $\mathbf{\pm S D}$ & Mean & $\mathbf{\pm S D}$ & P-value \\
\hline Age (years) & 28 & 6 & 28 & 8 & 29 & 7 & 0.796 \\
Duration of surgery (min) & 54 & 18 & 63 & 20 & 63 & 17 & 0.087 \\
Weight (kg) & 75 & 16 & 79 & 14 & 80 & 10 & 0.332 \\
Height $(\mathbf{c m})$ & 162 & 7 & 161 & 5 & 163 & 6 & 0.713 \\
\hline
\end{tabular}

One-way ANOVA test was used

Table (2) $\mathrm{SPO}_{2}$ in the study groups at different times.

\begin{tabular}{lccccccc}
\hline & Group A & $(\mathbf{n}=\mathbf{3 0})$ & \multicolumn{2}{c}{ Group B $(\mathbf{n}=\mathbf{3 0})$} & \multicolumn{2}{c}{ Group C $(\mathbf{n}=\mathbf{3 0})$} & \\
& Mean & $\mathbf{\pm S D}$ & Mean & $\mathbf{\pm S D}$ & Mean & $\mathbf{\pm S D}$ & P-value \\
\hline Baseline & 98 & 1 & 98 & 1 & 98 & 1 & 0.313 \\
At 15 minutes & 98 & 1 & 98 & 1 & 98 & 1 & 1.0 \\
At 30 minutes & 98 & 1 & 98 & 1 & 98 & 1 & 0.591 \\
At 45 minutes & 98 & 1 & 98 & 1 & 98 & 1 & 0.12 \\
At 60 minutes & 98 & 1 & 98 & 1 & 98 & 1 & 0.108 \\
At 75 minutes & 98 & 1 & 98 & 1 & 99 & 1 & 0.126 \\
At 90 minutes & 98 & 1 & 99 & 1 & 99 & 1 & 0.264 \\
\hline
\end{tabular}

One-way ANOVA test was used

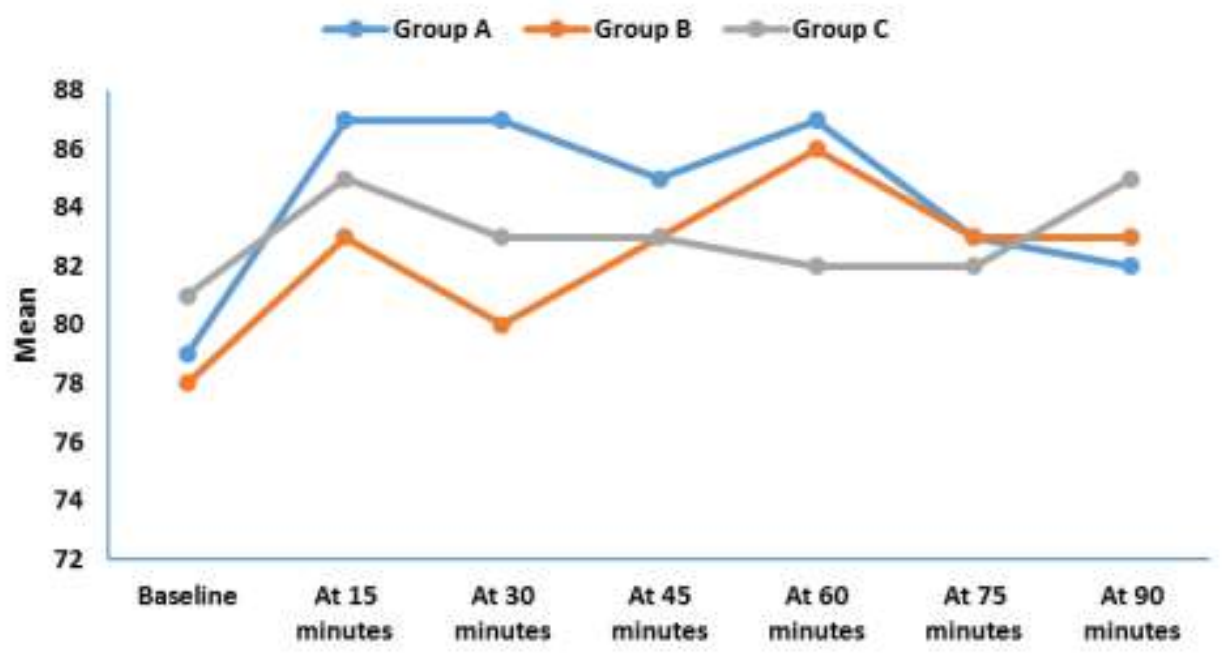

Fig. (1) Heart rate in the study groups at different times. 
EtCO2 showed no significant differences between the three groups at different time points.

The VAS score at rest showed an overall significant difference between the three groups starting from 4 hours. The Median VAS score was significantly higher in group A than B \& C at 4, 6, 12, and 24 hours. Also, it was significantly higher in group $\mathrm{C}$ than group $\mathrm{B}$ at all these times except at 12 hours, as no significant difference was reported between groups B \& C at this time. These data are shown in fig. (3).

VAS score during cough showed an overall significant difference between the three groups starting from 2 hour. Median VAS score was significantly higher in group $\mathrm{A}$ than $\mathrm{B} \& \mathrm{C}$, with no differences between B \& C groups at 2, 6, 12, and 24 hours. While at 4 hours, VAS score was significantly lower in group $\mathrm{B}$ compared to group $\mathrm{A}$ and $\mathrm{C}$ with no differences between A \& C groups, fig. (4).

Rescue analgesia started at 2 hours. It was significantly lower in group B at all times than groups A \& C except at 12 hours it was lower in $C$ than A \&B with no significant differences between groups B \& C, fig. (5).

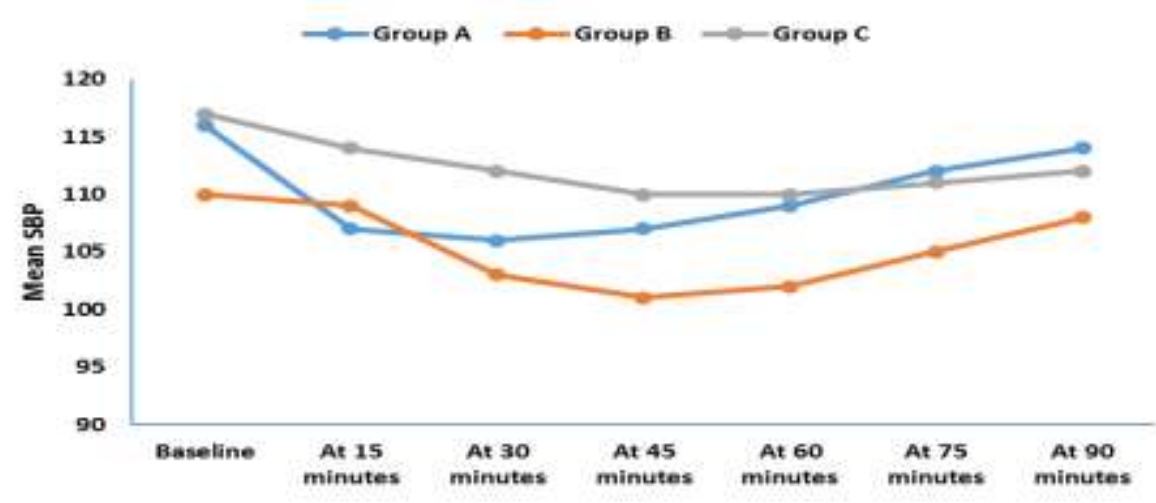

Fig. (2) Mean blood pressure in the study groups at different times.

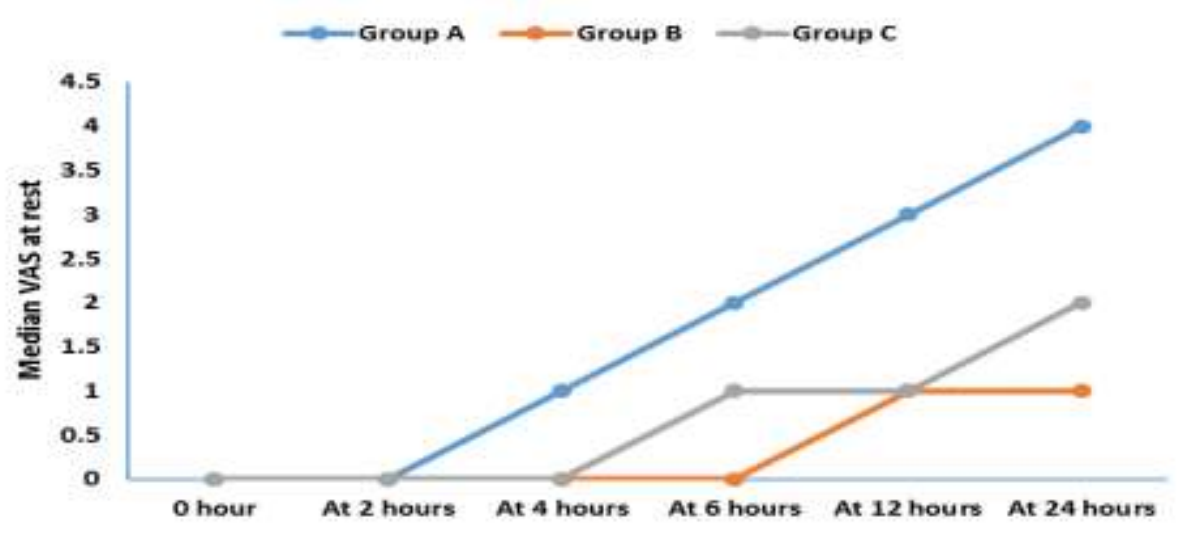

Fig. (3) VAS score at rest in the study groups at different times.

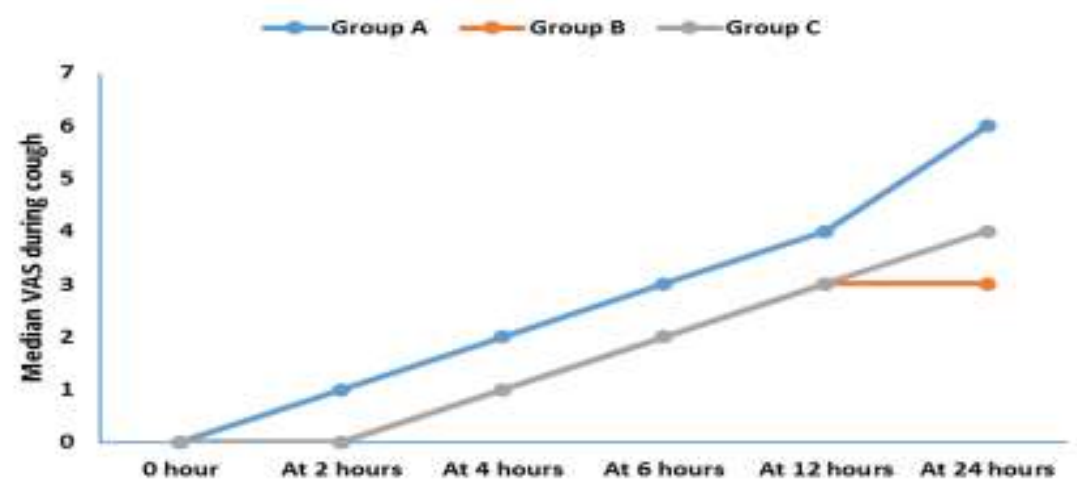

Fig. (4) VAS score during cough in the study groups at different times. 
Vomiting score showed an overall significance between the three groups. It showed that nausea and vomiting was significantly higher in group B than group A,C . No significant differences were found between groups A \& C. fig. (6)
Sedation scores at 2, 4, and 6 hours showed overall significant differences between the three groups. It revealed that sedation score was significantly lower in group B at all these times than groups A \& C, with no significant differences between groups A \& C. These data are shown in fig. (7).

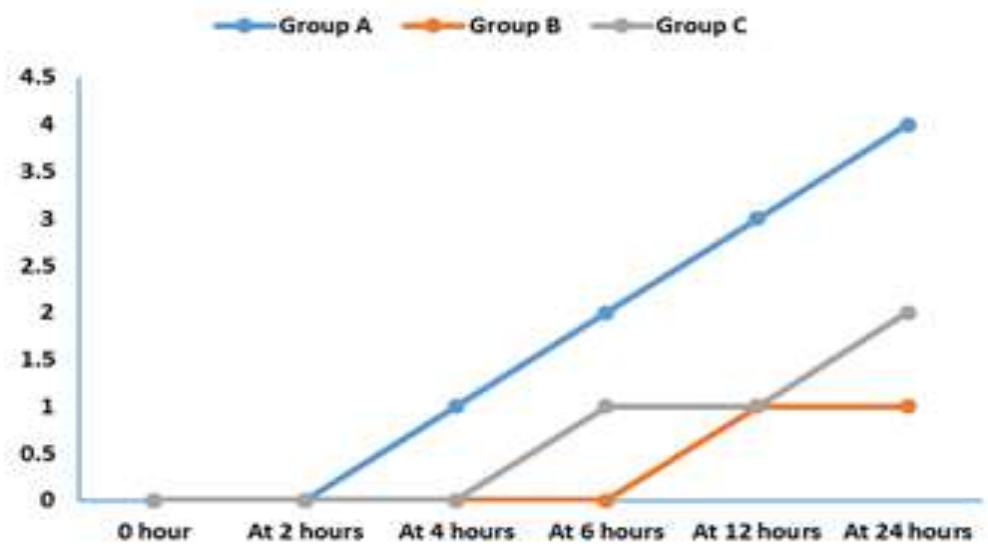

Fig. (5) Time To First Analgesia in the study groups at different times.

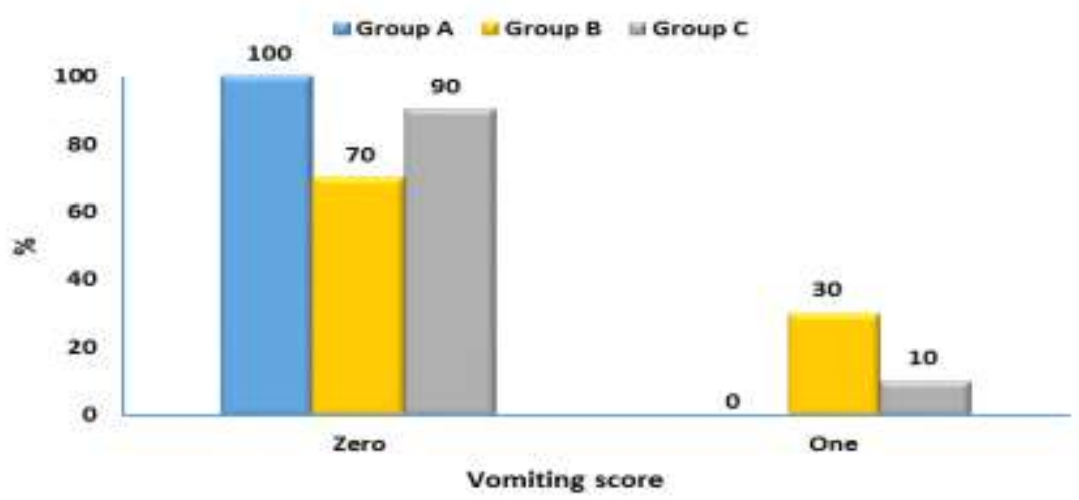

Fig. (6) Vomiting score in the study groups at different times.

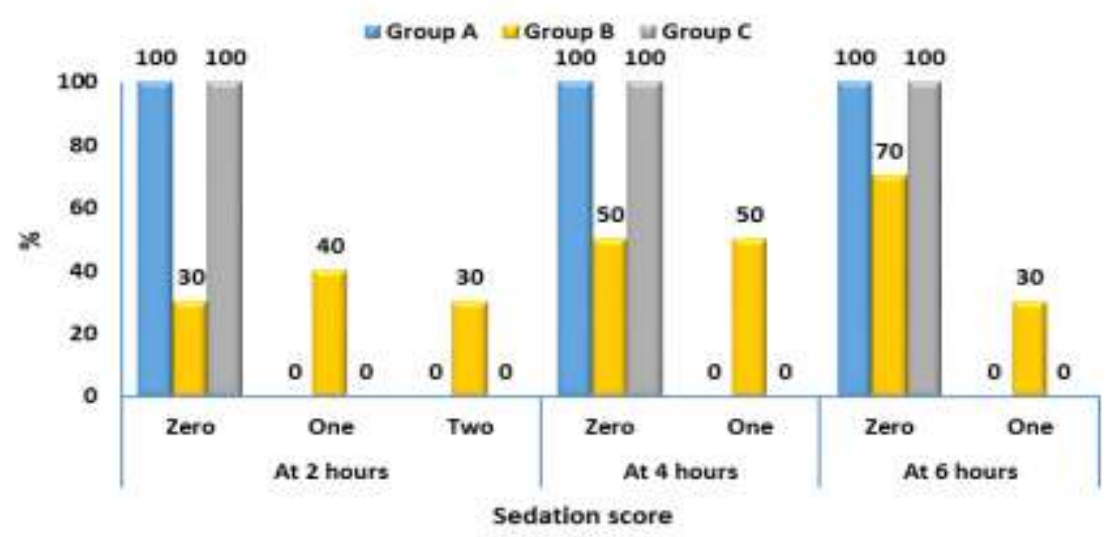

Fig. (7) Sedation score in the study groups at different times. 


\section{Discussion}

This research compares the effectiveness of decamedetomidine and dexamethasone as local anaesthetic supplements with the USG TAP guided block for post-operative caesarean analgesia. The present research has shown that dexmedetomidine is substantially reduced to bupivacaine in the TAP block in the first 12 hours, more sedative and prolonged TMA than dexamethasone.

The mechanisms underlying the analgesic action of dexmedetomidine are still not apparent and may be multifactorial in Myeong et al.[6] in their research on the administration of dexmedetomidine as an adjuvant to local anaesthetics.

In their research of dexamethasone as a levobupivacane adjuvant in the US, Akkaya et al [7] found that the mechanism of action still has to be studied. Another explanation of Myeong et al., Movafegh et al. and Bastos et al. was that the vasoconstriction may be related to the level perineal, resulting in slow absorption of local anaesthetics, suppression of synthesis and secretion of inflammatory mediators, reduction of unmyelinated C-fiber transmission, by immune suppressive effect or local action of the nerve. Some writers think that dexamethasone's analgesic effects have a systemic impact.

As regards dexametomemidine adjuvants, Thakur et al., have [8] reported that dexamedetomidine $1 \mu \mathrm{g} / \mathrm{kg}$ was almost the ideal dose as an adjuvant for axillary blocks, which increases post-operative analgesic duration and delays the requirement of the first dose of analgesics, and this was the dose of Zhang et al.

[10] Meta-analysis Qi Chen et al. showed that perineural dexamethasone extended the LA block duration. In addition, administration of perineural dexamethasone was related with reduced painkiller consumption and lower nausea and vomiting incidence on the first day after surgery.

On the other hand, Myeong et al[6] demonstrated the combination of dexamethasone and dexmedetomidine to prolong the duration of ropivacaine in the ultrasonic brachial plexus plexus block with nerve stimulation in their research. This may be due to the ropivacaine use instead of bupivacane since ropivacaine has a longer action time and the effect of adjuvants may not be apparent because the grade of sensory block is evaluated by pins based on a two-grade scale $(0=$ no block, $1=$ loss of sensation of pinprick) and not VAS as in this study.

In the VAS score, Magdy et al.[11] repository and movement ratings were much lower in the TABD group compared with the TAP and LAI groups. TAP block with dexmedetomidine showed a reduced range of VAS discomfort after 10, 18 and 24 hours of rest and movement.

From 4 hours, the remainder of the VAS score revealed an overall significant difference between the three groups (P-value was $<0.001$ each time). In this research Post-hoc analyses showed that the median
VAS score in groups A was significantly higher at 4, 6, 12 , and 24 hours than B\&C. In group C, it was much greater at all periods than group $\mathrm{B}$ with the exception of 12 hours, because no significant difference was observed between groups B \& C at this time. The overall significant difference between the three groups was seen in the VAS scoring during cough (P-value was $<0.001$ per time). Post hoc analyses showed that the median VAS ratings in Group A were much higher at 2, 6, 12 and 24 hours, and there were no differences between B\&C groups. At 4 hours, the VAS score in group B was substantially lower than in group A and C, while there were no differences between A \& $\mathrm{C}$ groups.

We observed that there were no statistically significant differences in the first 24 hours between the three groups in the total cumulative dosage of morphine after surgery.

The findings were not statistically significantly different from nausea and vomiting. This is contradictory to Ammar et alfindings, .'s which showed that the incidence of nausea and vomiting was reduced in their dexamethasone trial. The discrepancy with Ammar et al. [12] may arise from comparing dexamethasone to placebo in their research, or from antiemetic being the premedicated drug that we used in their trial before anaesthesia was induced.

The outcome of the sedation score was statistically significantly different (with $\mathrm{p}<0.001$ ) and was persistent in favour of dexmedetomidine B for 6 hours after surgery since this induces sedation while dexamethasone has no sedating effect.

In all three groups [group A, B, C] the time for first-aid analgesia was similar, with most of our patients receiving the initial rescue analgesia in the first two hours. Probably because of the visceral discomfort, because the somatic pain caused by the incision was insignificant. TAP single injection protects against somatic pain, while visceral discomfort is avoided. TAP block inhibits the neuronal afferents of the abdomen wall between T6-L1. The pain in caesarean section basically includes two somatic and visceral parts, although the abdominal wall incision leads to significant discomfort in patients. Using the USG guided TAP block for caesarean region, a randomised double-blind Uma Srivastava et.al studies showed that the main somatic pain was extremely easy to treat with the TAP block and visceral discomfort at worst didn't seem significant and was supplemented with more analgesics. [1]

In her paper, Ralph et al. reported that dexmedetomidine's major sedative and anti-social effects are due to its stimulation of the $\alpha 2$ adrenoceptors in the locus coeruleus, which is the predominant noradrenergic nucleus in the brain and an important modulator of alertness, and is also the site of the downward medullospinal noradrenergies known as the impo [10]

Furthermore, investigations in transgenic mice showed that the dexmedetomidin's sedative and analgesic effects are relayed via an $\alpha 2 \mathrm{~A}$-adrenoceptor 
subtype. The enhanced specificities of dexmedetomidine for the $\alpha 2$ receptor, in particular for this receptor's $2 \mathrm{~A}$ subtype, make it a considerably more efficient sedative and painkiller than clonidine [10].

\section{Conclusion}

Our research has shown that adding dexmedetomidine to the TAP block substantially reduces the value of VAS, less drug usage during the first 12 hours, greater sedation and prolonged TFA than dexamethasone.

\section{References}

[1] U. Srivastava. "Efficacy of trans abdominis plane block for post cesarean delivery analgesia: A double-blind, randomized trial," Saudi J. Anaesth., vol. 9, no. 3, p. 298, 2015.

[2] A. E. Farhat, M. E. Elkafrawy, and D. M. Effat, "TAP Block with dexamethasone as a Pain Treatment Modality after Lower Abdominal Gynecological Procedures," Al-Azhar Int. Med. J., vol. 23, pp. 23-31, 2021.

[3] A. A.R.Metwally, K. M. Abo-El-Enin, S. I. Abd Allah, N. M. Soliman, and W. A. Abo-Omar, "Ultrasound-guided transversus abdominis plane block for lower abdominal surgeries: bupivacaine alone or combined with fentanyl or epinephrine," Menoufia Med. J., vol. 30, pp. 538, 2017.

[4] D. Hewson, N. Bedforth, C. McCartney, and J. Hardman, "Dexamethasone and peripheral nerve blocks: back to basic (science)," Br. J. Anaesth., vol. 122, pp. 411-412, 2019.

[5] K. An, N. M. Elkassabany, and J. Liu, "Dexamethasone as adjuvant to bupivacaine prolongs the duration of thermal antinociception and prevents bupivacaine-induced rebound hyperalgesia via regional mechanism in a mouse sciatic nerve block model," PLoS One, vol. 10, pp. e0123459, 2015.

[6] M. J. Lee, D. J.Koo, Y.S.Choi, K.C.Lee, and H.Y.Kim, "Dexamethasone or dexmedetomidine as local anesthetic adjuvants for ultrasound-guided axillary brachial plexus blocks with nerve stimulation," Korean J. Pain, vol. 29, pp. 29, 2016.

[7] A. Akkaya "Dexamethasone added to levobupivacaine in ultrasound-guided tranversus abdominis plain block increased the duration of postoperative analgesia after caesarean section: a randomized, double blind, controlled trial," Eur Rev Med Pharmacol Sci, vol. 18, pp. 717722,2014

[8] A. Thakur, J. Singh, S. Kumar, S. Rana, P.Sood, and V.Verma, "Efficacy of dexmedetomidine in two different doses as an adjuvant to lignocaine in patients scheduled for surgeries under axillary block," J. Clin. diagnostic Res. JCDR, vol. 11, pp. UC16, 2017.
[9] Y. Zhang ."Perineural administration of dexmedetomidine in combination with ropivacaine prolongs axillary brachial plexus block," Int. J. Clin. Exp. Med., vol. 7, no. 3, p. 680, 2014.

[10] Q. Chen, R. An, J. Zhou, and B. Yang, "Clinical analgesic efficacy of dexamethasone as a local anesthetic adjuvant for transversus abdominis plane (TAP) block: a meta-analysis," PLoS One, vol. 13, pp. e0198923, 2018.

[11] M. H. ELDEGWY and R. ALFIKEY, "UltrasoUnd-gUided taP Block with or withoUt dexmedetomidine VersUs local infiltration of the woUnd after oPen herniorrhaPhy: a randomized ProsPectiVe controlled stUdy." v.ol. 3, pp. 12-23, 2017

[12] A. S. Ammar and K. M. Mahmoud, "Effect of adding dexamethasone to bupivacaine on transversus abdominis plane block for abdominal hysterectomy: A prospective randomized controlled trial," Saudi J. Anaesth., vol. 6, pp. 229, 2012. 